OPEN ACCESS

Edited by:

Vincenzo Ciminale,

Università degli Studi di Padova, Italy

Reviewed by:

Steven Jacobson,

National Institute of Neurological

Disorders and Stroke (NINDS),

United States

Roberto S. Accolla,

University of Insubria, Italy

*Correspondence:

Fabio E. Leal

fabio.leal@inca.gov.br:

fabioit@me.com

Johan Van Weyenbergh

johan.vanweyenbergh@kuleuven.be:

j.vw@live.be

† Joint first authors.

Specialty section:

This article was submitted to

Virology,

a section of the journal

Frontiers in Microbiology

Received: 31 January 2018

Accepted: 26 April 2018

Published: 22 May 2018

Citation:

Leal FE, Menezes SM, Costa EAS, Brailey PM, Gama L, Segurado AC,

Kallas EG, Nixon DF, Dierckx T,

Khouri R, Vercauteren $J$,

Galvão-Castro B, Raposo RA and

Van Weyenbergh J (2018)

Comprehensive Antiretroviral

Restriction Factor Profiling Reveals the

Evolutionary Imprint of the ex Vivo and

in Vivo IFN- $\beta$ Response in

HTLV-1-Associated

Neuroinflammation.

Front. Microbiol. 9:985.

doi: 10.3389/fmicb.2018.00985

\section{Comprehensive Antiretroviral} Restriction Factor Profiling Reveals the Evolutionary Imprint of the ex Vivo and in Vivo IFN- $\beta$ Response in HTLV-1-Associated Neuroinflammation

Fabio E. Leal ${ }^{1,2 * t}$, Soraya Maria Menezes ${ }^{3 \dagger}$, Emanuela A. S. Costa ${ }^{4}$, Phillip M. Brailey ${ }^{1}$, Lucio Gama ${ }^{5}$, Aluisio C. Segurado ${ }^{4}$, Esper G. Kallas ${ }^{4}$, Douglas F. Nixon ${ }^{1}$, Tim Dierckx ${ }^{3}$, Ricardo Khouri ${ }^{3,6}$, Jurgen Vercauteren ${ }^{3}$, Bernardo Galvão-Castro ${ }^{7}$, Rui Andre Saraiva Raposo ${ }^{1}$ and Johan Van Weyenbergh ${ }^{3 *}$

${ }^{1}$ Oncovirology Program, Instituto Nacional de Câncer (INCA), Rio de Janeiro, Brazil, ${ }^{2}$ Microbiology Immunology and Tropical Medicine, George Washington University, Washington, DC, United States, ${ }^{3}$ Department of Microbiology and Immunology, Rega Institute for Medical Research, KU Leuven, Leuven, Belgium, ${ }^{4}$ Departamento de Moléstias Infecciosas e Parasitárias, Faculdade de Medicina, Universidade de São Paulo, São Paulo, Brazil, ${ }^{5}$ Department of Molecular and Comparative Pathobiology, Johns Hopkins University School of Medicine, Baltimore, MD, United States, ${ }^{6}$ Fundação Oswaldo Cruz, Instituto Gonçalo Moniz (IGM), Salvador-Bahia, Brazil, ${ }^{7}$ Escola Bahiana de Medicina e Saúde Pública, Salvador-Bahia, Brazil

HTLV-1-Associated Myelopathy (HAM/TSP) is a progressive neuroinflammatory disorder for which no disease-modifying treatment exists. Modest clinical benefit from type I interferons (IFN- $\alpha / \beta)$ in HAM/TSP contrasts with its recently identified IFN-inducible gene signature. In addition, IFN- $\alpha$ treatment in vivo decreases proviral load and immune activation in HAM/TSP, whereas IFN- $\beta$ therapy decreases tax mRNA and lymphoproliferation. We hypothesize this "IFN paradox" in HAM/TSP might be explained by both cell type- and gene-specific effects of type I IFN in HTLV-1-associated pathogenesis. Therefore, we analyzed ex vivo transcriptomes of $\mathrm{CD}^{+}{ }^{+} \mathrm{T}$ cells, PBMCs and whole blood in healthy controls, HTLV-1-infected individuals, and HAM/TSP patients. First, we used a targeted approach, simultaneously quantifying HTLV-1 mRNA (HBZ, Tax), proviral load and 42 host genes with known antiretroviral (anti-HIV) activity in purified $\mathrm{CD}^{+}{ }^{+} \mathrm{T}$ cells. This revealed two major clusters ("antiviral/protective" vs. "proviral/deleterious"), as evidenced by significant negative (TRIM5/TRIM22/BST2) vs. positive correlation (ISG15/PAF1/CDKN1A) with HTLV-1 viral markers and clinical status. Surprisingly, we found a significant inversion of antiretroviral activity of host restriction factors, as evidenced by opposite correlation to in vivo HIV-1 vs. HTLV-1 RNA levels. The anti-HTLV-1 effect of antiviral cluster genes was significantly correlated to their adaptive chimp/human evolution score, for both Tax mRNA and PVL. Six genes of the proposed antiviral cluster underwent lentivirus-driven purifying selection during primate evolution (TRIM5/TRIM22/BST2/APOBEC3F-G-H), underscoring the cross-retroviral evolutionary imprint. Secondly, we examined the genome-wide type I IFN response in HAM/TSP 
patients, following short-term ex vivo culture of PBMCs with either IFN- $\alpha$ or IFN- $\beta$. Microarray analysis evidenced 12 antiretroviral genes (including TRIM5 $\alpha / T R I M 22 / B S T 2$ ) were significantly up-regulated by IFN- $\beta$, but not IFN- $\alpha$, in HAM/TSP. This was paralleled by a significant decrease in lymphoproliferation by IFN- $\beta$, but not IFN$\alpha$ treatment. Finally, using published ex vivo whole blood transcriptomic data of independent cohorts, we validated the significant positive correlation between TRIM5, TRIM22, and BST2 in HTLV-1-infected individuals and HAM/TSP patients, which was independent of the HAM/TSP disease signature. In conclusion, our results provide ex vivo mechanistic evidence for the observed immunovirological effect of in vivo IFN$\beta$ treatment in HAM/TSP, reconcile an apparent IFN paradox in HTLV-1 research and identify biomarkers/targets for a precision medicine approach.

Keywords: HTLV-1, HIV, retrovirus, evolution, interferon, neuroinflammation, multiple sclerosis, transcriptomics

\section{BACKGROUND}

Human T-cell Lymphotropic Virus-1 (HTLV-1), recently renamed Human T-cell Leukemia Virus-1 due to its strong oncogenic potential (Gallo et al., 2017; Tagaya and Gallo, 2017), is also the causative agent of the debilitating neuroinflammatory disorder, HTLV-1-Associated Myelopathy/Tropical Spastic Paraparesis (HAM/TSP) (Osame et al., 1986). HAM/TSP is associated with high HTLV-1 proviral load (PVL) and transcriptional levels of retroviral regulatory genes, Tax and HBZ (Saito et al., 2009). The pathogenesis of HAM/TSP is complex (Bangham et al., 2015), and a proportion of HTLV-1 asymptomatic carriers (AC) may present a PVL and inflammatory profile similar to HAM/TSP patients but do not develop clinical symptoms. It is unknown why 2$3 \%$ of HTLV-1 infected patients develop HAM/TSP after years of latent infection, but an interferon-inducible gene signature has been identified in HAM/TSP and is absent in AC (Tattermusch et al., 2012). However, type I interferonbased clinical trials reported modest clinical benefit, as well as antiviral (decreased PVL, Tax mRNA levels) and immunomodulatory effects (decreased T-cell spontaneous proliferation and activation), suggesting IFN- $\alpha$ and IFN- $\beta$ may hamper disease progression (Izumo et al., 1996; Oh et al., 2005). To reconcile this apparent contradiction, we hypothesize specific IFN-stimulated genes, e.g. antiviral effector genes, can exert deleterious vs. protective roles in HAM/TSP, as we recently suggested for B-cell expression of CD80 and CD86 (Menezes et al., 2014).

In contrast to HTLV-1, several anti(retro)viral effector genes have been classified as "restriction factors" (RFs) for HIV-1 infection, some of which are modulated by type I IFNs (Foster et al., 2017). We have previously compiled and validated a realtime PCR-array of 42 well-characterized RFs with suppressive activity against HIV-1 (Neil et al., 2008; Abdel-Mohsen et al., 2013; Raposo et al., 2013a,b, 2014), including APOBECs, TRIMs, and BST2/Tetherin.. Enhanced HIV-1 infection upon siRNA-mediated silencing in neuroblasts illustrates the relevance of TRIM5 $\alpha$ and TRIM22 in neuroinfection (Singh et al., 2014).
To assess the role of antiretroviral genes in HAM/TSP pathogenesis, we determined transcriptional levels of $42 \mathrm{RFs}$ and regulatory HTLV-1 genes Tax and $\mathrm{HBZ}$ in $\mathrm{CD}^{+} \mathrm{T}$ cells from HTLV-1 patients. We identified a strong negative association between expression of HTLV-1 Tax and a cluster of RFs, including TRIM5 $\alpha /$ TRIM22/BST2. Genome-wide transcriptomic analysis of HAM/TSP patients showed a significant and distinct up-regulation of RFs after ex vivo exposure to IFN- $\beta$, but not IFN- $\alpha$. These results provide mechanistic evidence for the immunovirological impact of IFN- $\beta$ therapy observed in vivo in HAM/TSP patients and pave the way to an evidence-based precision medicine approach to this neuroinflammatory disorder.

\section{PATIENTS AND METHODS}

All participants signed a written informed consent in accordance with the Declaration of Helsinki, approved by the University Institutional Review Boards of USP (\#0855/08) and CPqGMFIOCRUZ (\#022/03) before inclusion in the study. Ex vivo analysis of antiretroviral restriction factors was conducted in a cohort of 18 individuals (7 HAM/TSP, 6 HTLV-1-infected asymptomatic carriers (AC) and 5 age- and gender-matched HTLV-1-negative healthy controls (HC)), enrolled at the HTLV1 Outpatient Clinic at the University of Sao Paulo (USP), Brazil. The second cohort (lymphoproliferation/microarray analysis) consisted of $10 \mathrm{HAM} / \mathrm{TSP}$ patients, followed at the Bahiana School of Medicine and Public Health HTLV reference center in Salvador-Bahia, Brazil. Validation of transcriptomic findings was performed in a third, published cohort (London, UK). Demographic and clinical data of all cohorts are detailed in Table 1. Clinical status was determined based on WHO criteria for HTLV-1 associated diseases (Osame, 1990). None of the HAM/TSP patients had received prior IFN-based therapy. Blood samples were obtained and processed with Ficoll-Paque PLUS (Amersham Pharmacia Biotech, Uppsala, Sweden) gradient centrifugation and peripheral-blood mononuclear cells (PBMC) were isolated and either cryopreserved in $10 \%$ DMSO in FBS (RF analysis) or used for short-term ex vivo culture (48 h for microarray analysis, $96 \mathrm{~h}$ for lymphoproliferation), in the absence 
TABLE 1 | Demographic and clinical data of 3 HTLV-1 cohorts.

\begin{tabular}{|c|c|c|c|c|}
\hline Cohorts & $\mathrm{HC}$ & AC & HAM/TSP & Cell type \\
\hline $\begin{array}{l}\text { Cohort } 1 \text { (Sao } \\
\text { Paulo, BR) }\end{array}$ & & & & CD4+ T cells \\
\hline $\begin{array}{l}\text { Number of } \\
\text { samples }\end{array}$ & 5 & 6 & 7 & \\
\hline $\begin{array}{l}\text { Age (median, } \\
\text { IQR) }\end{array}$ & $54(38-61)$ & $62(54-66)$ & $51(45-62)$ & \\
\hline Gender & $1 \mathrm{M} / 4 \mathrm{~F}$ & $1 \mathrm{M} / 5 \mathrm{~F}$ & $2 \mathrm{M} / 5 \mathrm{~F}$ & \\
\hline $\begin{array}{l}\text { PVL (median, } \pm \\
\text { SD) }\end{array}$ & N/A & $7.6 \pm 151.5$ & $181.0 \pm 134.5$ & \\
\hline $\begin{array}{l}\text { Tax mRNA } \\
\text { (median, } \pm \text { SD) }\end{array}$ & N/A & $0.02 \pm 0.02$ & $0.68 \pm 0.77$ & \\
\hline $\begin{array}{l}\text { HBZ mRNA } \\
\text { (median, } \pm \text { SD) }\end{array}$ & $\mathrm{N} / \mathrm{A}$ & $10^{-5} \pm 5.410^{-}$ & $2.710^{-4} \pm 8.110^{-4}$ & \\
\hline $\begin{array}{l}\text { Cohort } 2 \\
\text { (Bahia, BR) }\end{array}$ & & & & PBMC \\
\hline $\begin{array}{l}\text { Number of } \\
\text { samples }\end{array}$ & 11 & 5 & 10 & \\
\hline $\begin{array}{l}\text { Age (median, } \\
\text { IQR) }\end{array}$ & & & $52 \pm 6.2$ & \\
\hline Gender & $4 \mathrm{M} / 7 \mathrm{~F}$ & $2 \mathrm{M} / 3 \mathrm{~F}$ & $4 \mathrm{M} / 6 \mathrm{~F}$ & \\
\hline $\begin{array}{l}\text { Cohort } 3 \\
\text { (published; } \\
\text { London, UK) }\end{array}$ & & & & Whole blood \\
\hline $\begin{array}{l}\text { Number of } \\
\text { samples }\end{array}$ & 8 & 17 & 10 & \\
\hline $\begin{array}{l}\text { Age (median, } \\
\text { IQR) }\end{array}$ & $49(46-71)$ & $47(38-62)$ & 54(49-62) & $63(44-74)$ \\
\hline Gender & $4 \mathrm{M} / 4 \mathrm{~F}$ & $0 M / 17 F$ & $4 \mathrm{M} / 6 \mathrm{~F}$ & \\
\hline $\begin{array}{l}\text { PVL (median, } \\
\text { IQR) }\end{array}$ & N/A & $0.6(0.4-0.9)$ & $3.9(3.4-6.7)$ & $10.1(6.7-17.8)$ \\
\hline $\begin{array}{l}\text { Total number of } \\
\text { individuals }\end{array}$ & 24 & 23 & 27 & \\
\hline
\end{tabular}

or presence of $1,000 \mathrm{IU} / \mathrm{ml}$ clinical grade IFN- $\alpha$ (gift of Blausiegel Ltda., Sao Paulo-Brazil) or IFN- $\beta$ (gift of Dr. Brassat, ToulouseFrance), as previously described (Moens et al., 2012a; Dierckx et al., 2017).

\section{Proviral Load and mRNA Assessment}

Total DNA and RNA were extracted from enriched CD $4^{+}$T cells using a commercial kit (Qiagen GmbH, Hilden Germany) and cDNA was generated using Superscript VILO cDNA synthesis kit (Invitrogen) following the manufacturer's instructions. HTLV-1 proviral load absolute quantification was performed as previously described (Dehee et al., 2002), normalized to human albumin gene. Samples were assayed in duplicate. HTLV-1 proviral load was calculated as follows: copy number of HTLV-1 per 1,000 $\mathrm{CD}^{+}{ }^{+} \mathrm{T}$ cells $=($ copy number of HTLV-1) $/$ (copy number of albumin) $\times 2 \times 1,000$ cells.

Transcription levels of Tax and HBZ were measured as previously described (Saito et al., 2009), using housekeeping gene Ubiquitin C (Life Technologies assay ID\# Hs00824723_m1) to calculate tax and HBZ $2^{-\Delta \mathrm{Ct}}$ relative expression. Relative quantification of 42 RFs using
mRNA from $\mathrm{CD}^{+}{ }^{+} \mathrm{T}$ cells from $13 \mathrm{HTLV}$-1-infected patients and 5 uninfected healthy controls was performed using custom-made $\operatorname{TaqMan}^{\circledR}$ Low Density Arrays previously described (Abdel-Mohsen et al., 2013)(Applied Biosystems, Foster City, CA). RF gene descriptions are given in Table 2.

\section{Microarray Analysis}

Total RNA extraction (RNeasy kit, QIAGEN, Venlo, the Netherlands) and Whole Genome microarray analysis (HuGene 1.0 ST array, Affymetrix, Santa Clara, CA) were performed according to the manufacturers' specifications. Data were analyzed using the Bioconductor limma package. Microarray data used in this study are available at Gene Expression Omnibus under GEO accession number GSE82160 (Brazilian HTLV-1 cohort), GSE29333 (UK HTLV-1 cohort, Tattermusch et al.) and GSE18233 (Swiss HIV cohort, Rotger et al., 2010).

\section{Enrichment and Evolutionary Analysis}

Genome-wide enrichment analysis was performed using a modified Fisher's test, considering the total number of annotated genes for which transcripts were detectable by microarray ( $n$ $=22,370$ ), followed by stringent FDR (Benjamini-Hochberg) correction. Data and full methodology on human candidate retroviral restriction factors and chimp/human cross-species adaptive evolution and purifying selection during primate evolution are detailed in (Osame, 1990; Singh et al., 2014). In brief, measurements of cross-species adaptive evolution used the McDonald-Kreitman value to compare human sequence to chimpanzee in a set of 15,052 protein-coding genes (Singh et al., 2014) and purifying selection during primate evolution was measured using the proportion of nonsynonymous $\left(K_{\mathrm{A}}\right)$ over synonymous $\left(K_{\mathrm{S}}\right)$ substitutions per site in five primates (human, chimpanzee, orangutan, rhesus, and common marmoset) in a set of 140 candidate genes selected for their relationship to HIV pathogenesis (Osame, 1990).

\section{Statistical Analysis}

Statistical analysis was performed using GraphPad Prism software (GraphPad Software, version 6 and 7, San Diego, CA). Non-parametric statistical tests (Mann-Whitney, Wilcoxon tests, and Spearman correlation) were used for patient data, with Bonferroni correction for multiple comparisons between RFs, PVL and tax/HBZ mRNA levels. Transcriptome-wide correlation of mRNA expression levels with either set point viral load (HIV1) or TRIM5 (HTLV-1) mRNA expression was calculated using Spearman's correlation, followed by FDR correction for multiple testing.

\section{RESULTS}

\section{Antiretroviral RFs Separate in Two Major Clusters, Positively and Negatively Associated With HTLV-1 Virological and Clinical Status}

Based on a previously defined subset of host genes with significant anti-HIV activity (Abdel-Mohsen et al., 2013), we 
TABLE 2 | List of 42 antiretroviral genes measured in CD4+ ${ }^{+}$T cells.

\begin{tabular}{|c|c|}
\hline Target Information & NCBI Gene Description \\
\hline APOBECЗА-H & Apolipoprotein B mRNA editing enzyme, catalytic polipeptide-like 3 \\
\hline BST2/theterin & Bone Marrow Stromal cell antigen 2 \\
\hline SLFN11 & Schlafen family member 11 \\
\hline TRIM5 $\alpha$, TRIM11, TRIM21, TRIM22, TRIM26, TRIM28, TRIM32 & Tripartite motif family \\
\hline CPSF6 & Cleavage and Polyadenylation Specific Factor 6 \\
\hline SAMHD1 & SAM domain and HD domain 1 \\
\hline PML & Promyelocytic Leukemia protein \\
\hline RNF114 & Ring Finger Protein 114 \\
\hline Trex1 & Three Prime Repair Exonuclease 1 \\
\hline RPRD2 & Regulation of nuclear Pre-mRNA Domain containing 2 \\
\hline CHFR & Checkpoint with forkhead and ring finger domains, E3 ubiquitin protein ligase \\
\hline ISG15 & ISG15 ubiquitin-like modifier \\
\hline EIF2AK2/PKR & Eukaryotic translation initiation factor 2-alpha kinase 2 \\
\hline CTR9/PAF1/RTF1 & CTR9, PAF1, RTF1/RNA polymerase II complex component \\
\hline IFITM family (3 members) & Interferon induced transmembrane protein \\
\hline RSAD2 (viperin) & Radical S-adenosyl methionine domain containing 2 \\
\hline MOV10 & Moloney leukemia virus 10, homolog \\
\hline HERC5 & HECT domain and RLD 5 \\
\hline MX2 & MX dynamin like GTPase 2 \\
\hline CDKN1A & Cyclin-dependent kinase inhibitor $1 \mathrm{~A}$ \\
\hline BRD4 & Bromodomain containing 4 \\
\hline RNASEL & Ribonuclease L (2',5'-oligoisoadenylate synthetase-dependent) \\
\hline $\mathrm{CH} 25 \mathrm{H}$ & Cholesterol 25-Hydroxylase \\
\hline LGALS3BP & Lectin, Galactoside binding Soluble 3 Binding Protein \\
\hline CNP & $2^{\prime}, 3^{\prime}$-cyclic nucleotide $3^{\prime}$ phosphodiesterase \\
\hline IFl16 & Interferon gamma Inducible protein 16 \\
\hline
\end{tabular}

used a custom-made array to quantify the transcriptional levels of 42 RFs (listed in Table 2) in peripheral $\mathrm{CD}^{+}{ }^{+} \mathrm{T}$ cells from HTLV-1 infected patients or healthy controls. We compared the gene expression profiles of $13 \mathrm{HTLV}$-1-infected patients (6 AC, $7 \mathrm{HAM} / \mathrm{TSP}$ ) and 5 uninfected subjects using cluster analysis. As evident from Figure 1A, two major RF subsets appeared as separate clusters. First, a minor cluster of RFs (CDKN1A/ISG15 and PAF1) positively correlates with biomarkers of HTLV1 disease (PVL, mRNA levels of Tax and HBZ, as well as clinical status, box Figure 1A). Second, the largest cluster of RFs was found to correlate negatively with HTLV-1 biomarkers (Figure 1A). Within this large cluster, negative correlations between the expression levels of Tax and TRIM5 $\alpha(r=-0.86 ; p=$ $0.0084), \operatorname{TRIM} 22(r=-0.81 ; p=0.0336)$, BST2 $(r=-0.85 ; p=$ $0.0126)$, and RNASEL $(r=-0.83 ; p=0.021)$ were statistically significant after stringent Bonferroni correction (Figure 1B). Conversely, cyclin-dependent kinase inhibitor 1A (CDKN1A, also known as p21), a regulator of cell-cycle progression, was positively associated with HTLV-1 proviral load ( $r=0.84 ; p$ $=0.042$ ) (Figure 1C). Surprisingly, these ex vivo correlations recapitulate the in vivo findings of IFN- $\beta$ treatment in an immunovirological trial in HAM/TSP, in which IFN- $\beta$ was found to significantly decrease both tax mRNA levels and lymphoproliferation (Oh et al., 2005), but not PVL. In contrast, in vivo IFN- $\alpha$ treatment of HAM/TSP patients was found to decrease PVL and immune activation (Izumo et al., 1996). Therefore, we investigated if IFN- $\alpha / \beta$ might differentially affect lymphoproliferation and genome-wide expression profiles in HAM/TSP.

\section{Ex Vivo Lymphoproliferation Is Significantly Down-Regulated by IFN- $\beta$, but Not IFN- $\alpha$ in HAM/TSP}

We found that lymphoproliferation was significantly inhibited by IFN- $\beta(p<0.01)$, but not by IFN- $\alpha$ (Figure 2) This differential effect was not due to a defect in IFN- $\alpha$ bioactivity, since both IFNs displayed equal antiviral activity in a standardized Vesicular Stomatitis Virus (VSV) bioassay (Dierckx et al., 2017). This effect was specific for HAM/TSP, since no significant IFN-induced antiproliferative effect was observed in both healthy controls (Figure 2) and HTLV-1-infected carriers (data not shown).

\section{Genome-Wide Analysis Identifies Selective Up-Regulation of RFs by IFN- $\beta$, but Not IFN- $\alpha$, in HAM/TSP}

Since RFs can be IFN-regulated, we used microarray analysis to assess whether the proviral and antiviral clusters might be selectively up- or down-regulated by either IFN- $\alpha$ or IFN- $\beta$, the two IFN subtypes previously used in HAM/TSP trials (Izumo 
A

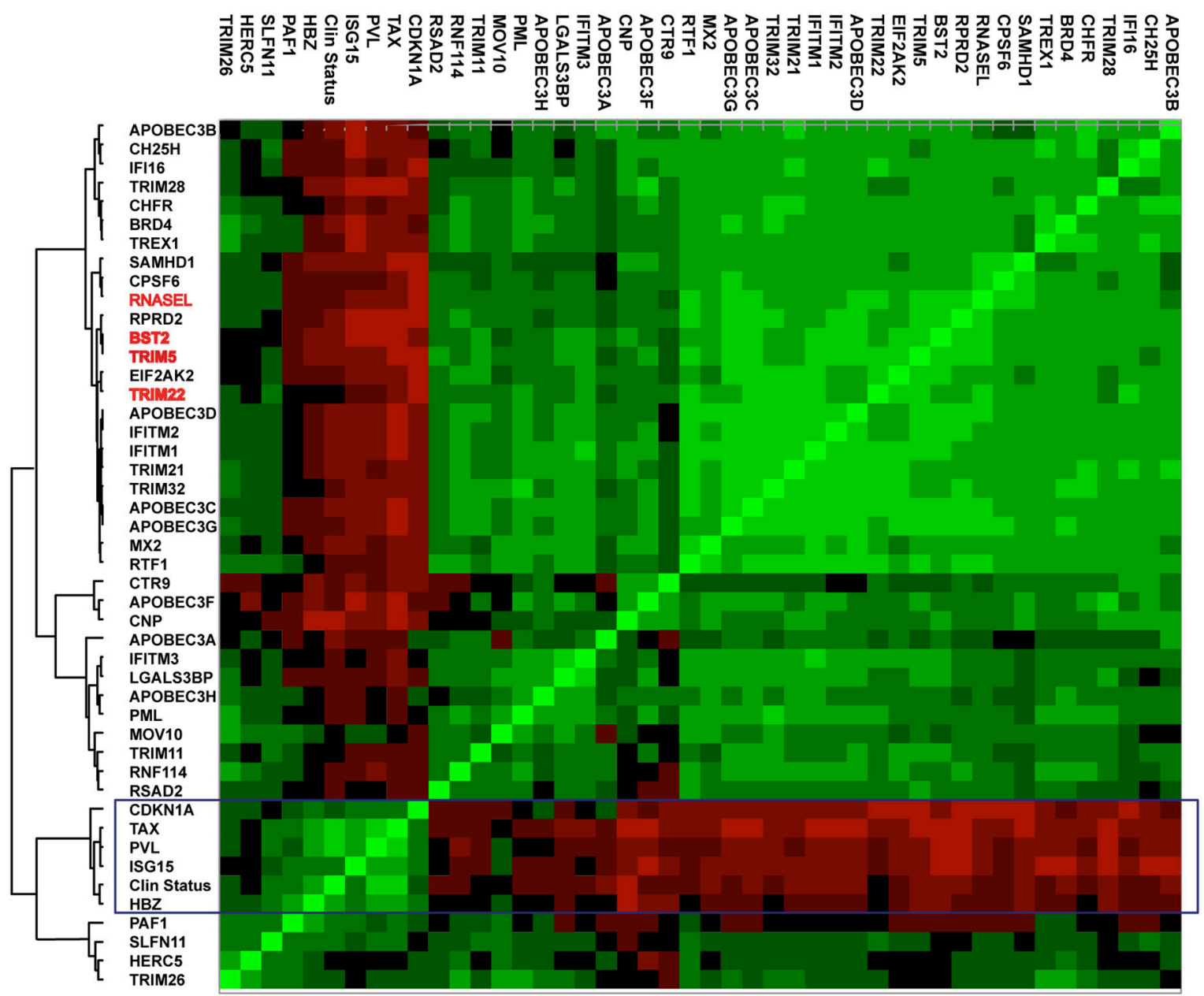

B

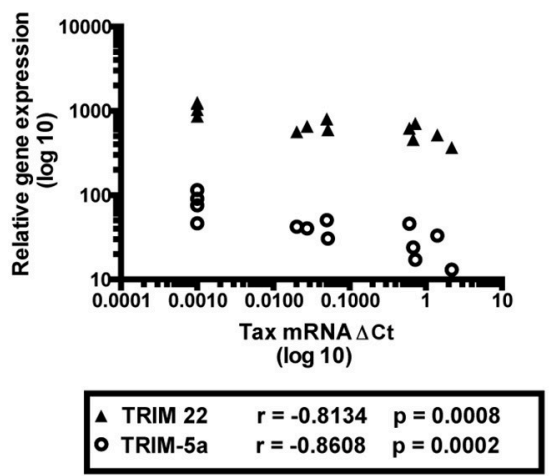

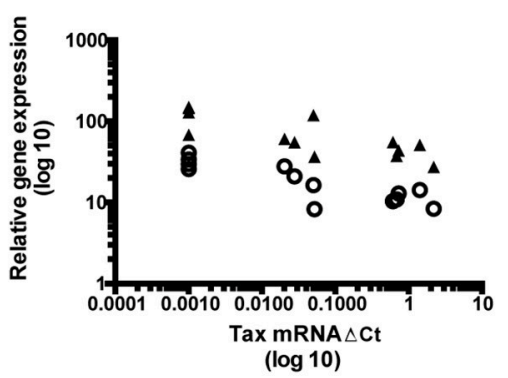

\begin{tabular}{|llcc|}
\hline BST-2 & BS $=-0.8497$ & $p=0.0003$ \\
0 & RNASEL & $r=-0.8329$ & $p=0.0005$ \\
\hline
\end{tabular}

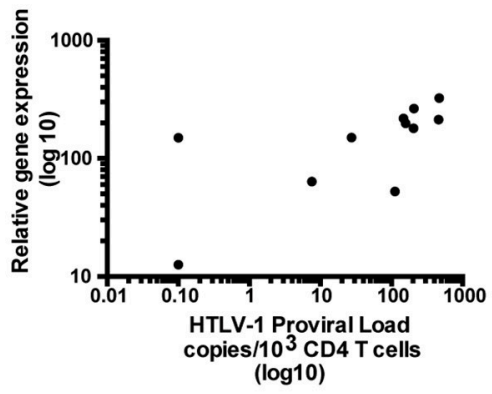

- CDKN1A $r=0.8427 \quad p=0.042$

FIGURE 1 | Association between host restriction factors (RFs) expression, HTLV-1 virological parameters and clinical status of HTLV-1 infected individuals. (A) Analysis of mRNA levels of 42 RFs, Tax/HBZ and HTLV- 1 proviral load (PVL) in CD4 ${ }^{+}$T cells from 6 asymptomatic carriers (AC), 7 HAM/TSP patients and 5 healthy donors (HD). Expression of host RFs was quantified by TLDA, results were clustered according to Spearman's correlation and shown as heatmap (red $=$ negative, green = positive). The boxed cluster indicates a minor subset of "proviral" RFs (CDKN1A, ISG15) clustering with HTLV-1 PVL, Tax mRNA, HBZ mRNA, and clinical status (HD/AC/HAM), whereas the majority of RFs forms a large "antiviral" cluster. A subset of antiviral RFs (TRIM5 $\alpha$, TRIM22, BST2, and RNASEL) with statistically significant negative correlation to tax mRNA are highlighted in red. (B) Correlation between TRIM5 $\alpha$, TRIM22, BST2, RNASEL, and tax mRNA (C) Correlation between CDKN1A and tax mRNA in HTLV-1-infected individuals ( $\mathrm{AC} n=6, \mathrm{HAM} n=7$ ). 


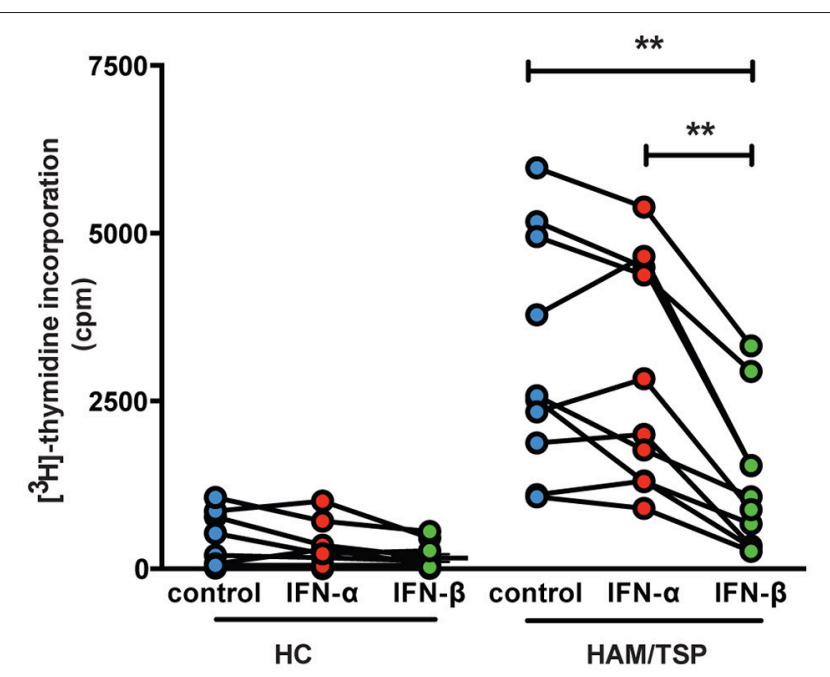

FIGURE 2 | Ex vivo lymphoproliferation is significantly down-regulated by IFN- $\beta$, but not IFN- $\alpha$ in HAM/TSP. Spontaneous lymphoproliferation was measured by $\left[{ }^{3} \mathrm{H}\right]$-thymidine incorporation, as previously described Moens et al. (2012a). The effect of IFN- $\alpha$ and IFN- $\beta$ (1000 IU/ml) upon ex vivo lymphoproliferation was determined in healthy controls $(\mathrm{HC}, n=11)$ and HAM/TSP patients $(n=10) .{ }^{* *} p<0.01$.

et al., 1996; Oh et al., 2005). As shown in Figure 3A, genomewide analysis identified 12/42 RFs (including TRIM $5 \alpha$, TRIM22, and BST2) were significantly up-regulated by IFN- $\beta(n=5$, enrichment $p<0.0001)$, vs. $0 / 42$ by IFN- $\alpha$ treatment $(n=6$, $p>0.05)$ during short-term $(48 \mathrm{~h})$ ex vivo culture of PBMC from HAM/TSP patients. In a striking parallel to our results in $\mathrm{CD}^{+} \mathrm{T}$ cells, where TRIM5 displayed the strongest negative correlation to Tax mRNA, TRIM5 was also identified as the top IFN- $\beta$-induced gene among the significant genes $(p=0.0009)$.

\section{Genome-Wide Analysis Identifies a Strongly Co-regulated TRIM5 $\alpha / T R I M 22 / B S T 2$ Subset in HTLV-1 Infection, Independent of the HAM/TSP Disease Signature}

To validate our findings, we analyzed published ex vivo whole blood transcriptomes in an additional HTLV-1-infected cohort (Tattermusch et al.; detailed in Table 1) (Osame et al., 1986). As shown in Figure 3B, significant positive correlations were confirmed between TRIM5 $\alpha /$ TRIM22 $(p=0.005, r=0.50)$ and TRIM5 $\alpha$ /BST2 $(p<0.001, r=0.67)$ in both HTLV-1-infected AC $(n=17)$ and HAM/TSP patients $(n=10)$. Of note, TRIM5 $\alpha$ mRNA expression was not significantly correlated to IFITM1 mRNA levels (data not shown), which is the only RF (among 42 studied herein) that is present in the HAM/TSP disease signature.

\section{RFs Correlate Oppositely to in Vivo Retroviral RNA Levels in Untreated HTLV-1} vs. HIV-1 Infection

Surprisingly, the "proviral/deleterious" gene cluster identified by its strong positive correlation to HTLV-1 RNA levels and
PVL contains the RFs previously demonstrated to exert a robust protective anti-HIV-1 effect in vivo and in vitro, namely CDKN1A, SLFN11, PAF1, and ISG15 (Telenti, 2005; Ortiz et al., 2009; McLaren et al., 2015; Nozuma et al., 2017). Therefore, we performed a pairwise analysis of correlation to in vivo RNA levels in both retroviral infections, using our HTLV-1 data (Figure 1A) and data from the Swiss HIV cohort (Rotger et al., 2010) by calculating transcriptome-wide correlation of viral load set point to each separate gene, followed by FDR correction. As shown in Figures 4A,B, a striking opposite effect can be observed for the "proviral" vs. "antiviral" clusters. HTLV-1 "antiviral" cluster genes show significantly increased (higher $R$-values) correlation to HIV-1 in vivo RNA levels ( $p<0.0001$, Wilcoxon signed rank test, Figure 4A). The reverse phenomenon can be observed for the HTLV-1 "proviral" cluster genes, showing a tendency for decreased (lower $R$-values) correlation to HIV-1 in vivo RNA levels ( $p=0.06$, Wilcoxon signed rank test, Figure 4B).

\section{Lentivirus-Driven Primate Evolution Has Shaped Antiretroviral Activity in Untreated HTLV-1 Infection}

Lentivirus infections, to which HIV and its ancestral SIV belong, have had a pronounced effect upon primate evolution (Telenti, 2005), whereas no similar evidence exists for deltaretroviruses, such as HTLV or PTLV. As shown in Figure 5, the antiviral RF cluster we identified for HTLV-1 is strongly selected during recent primate evolution, as measured by both crossspecies adaptive evolution between chimpanzees and humans (Figures 5A,B) and purifying selection throughout primate evolution (Figure 5C). Among the 42 anti-HIV RFs selected in this study, ten (TRIM5, APOBEC3A-B-C-F-G, IFI16, HERC5, EIF2AK2, and MX2) were found to display chimp/human adaptive evolution, as described by McLaren et al. (2015). The anti-HTLV-1 effect (measured as $R^{2}$ to represent effect size) of these 10 genes was significantly correlated to their adaptive evolution score, for both Tax mRNA $(r=0.86, p=0.0013$, Figure 5A) and PVL $(r=0.78, p=0.0073$, Figure 5B). In agreement with our results in Figures $1 \mathbf{A}, \mathbf{B}$, the effect size was greater for Tax mRNA (Figure 5A-range 0.1-0.74) than for PVL (Figure 5B-range 0.0-0.23), underscoring the putative role of these RFs in viral replication, rather than clonal expansion. Only six genes of the proposed "antiviral" cluster (TRIM5, TRIM22, and BST2, as well as APOBEC3F-G-H) underwent lentivirusdriven purifying selection during primate evolution (Ortiz et al., 2009), measured as $\mathrm{K}_{\mathrm{A}} / \mathrm{K}_{\mathrm{S}}$. In addition, these six HTLV-1correlated genes underwent a significantly stronger selection during primate evolution, as compared to all other lentivirusselected primate genes $(n=134, p<0.0001$, Mann-Whitney test, Figure 5C).

\section{DISCUSSION}

The striking negative association between mRNA levels of Tax and RFs TRIM5 $\alpha$, TRIM22, BST2, and RNASEL, despite the small number of participants in the study, suggests that these RFs may represent important mechanisms of defense against 

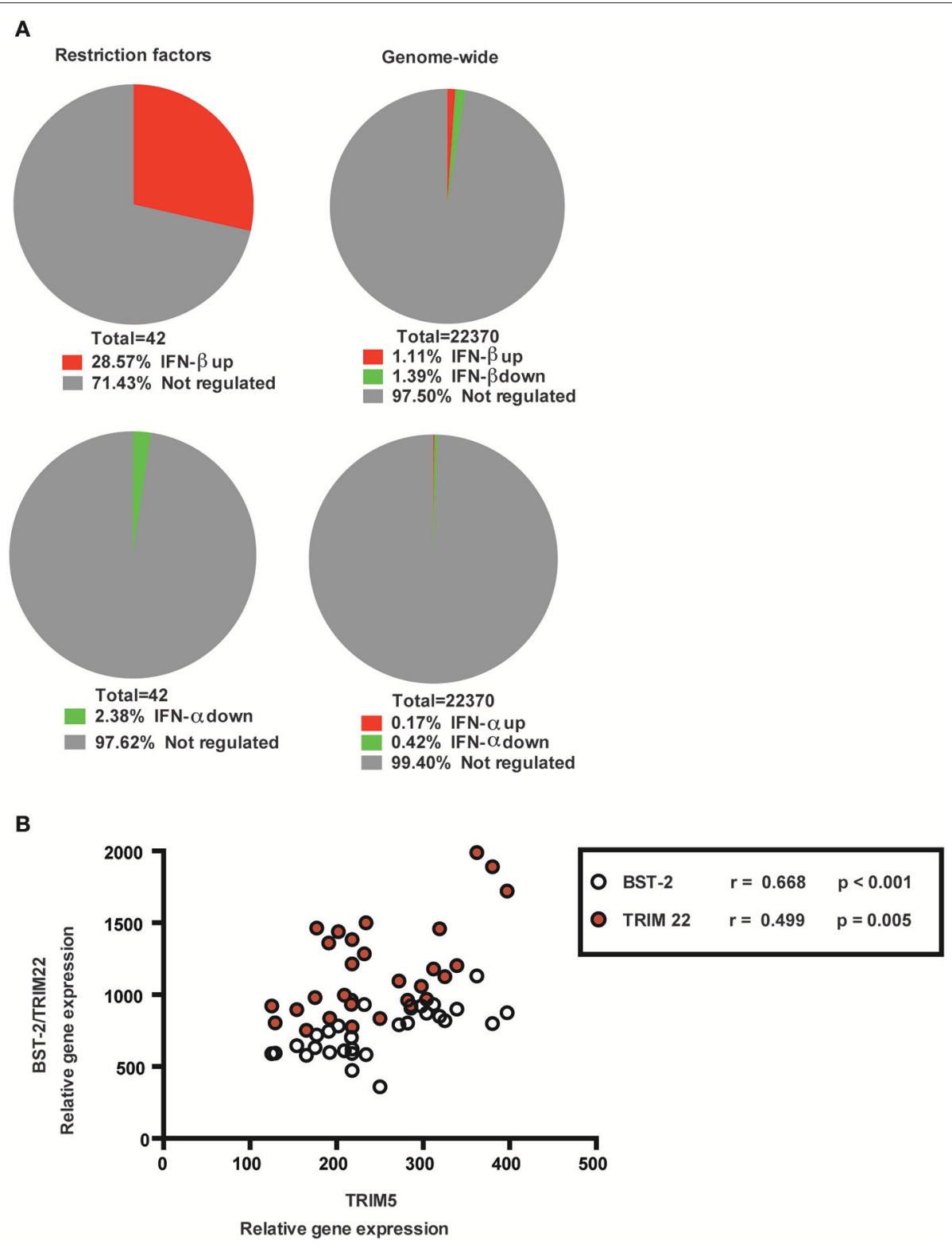

FIGURE 3 | Genome-wide analysis of PBMCs from HAM/TSP patients of RFs after ex vivo treatment with type I interferons. (A) Microarray analysis to determine genes significantly up- or down-regulated after $48 \mathrm{~h}$ ex vivo treatment of PBMCs from HAM/TSP patients with $1000 \mathrm{IU} / \mathrm{ml}$ IFN- $\beta$ ( $n=5$ ), upper right corner pie-chart or IFN- $\alpha(n=6)$, lower right corner pie-chart. RFs were significantly enriched among IFN- $\beta$-regulated, (upper left corner, genome-wide enrichment $p<0.0001)$, but not IFN- $\alpha$-regulated (lower left corner, $p>0.05$ ) genes. A total of 22370 genes were analyzed. (B) Reanalysis of published ex vivo whole blood transcriptomes from an independent HTLV-1 UK cohort (Tattermusch et al., 2012). Correlation between TRIM5 $\alpha$ and TRIM22/BST2 in both HTLV-1-infected AC ( $n=17$ ) and HAM/TSP patients $(n=10)$.

HTLV-1 infection and/or neuroinflammation. Our hypothesized antiviral activity of TRIM5 against HTLV-1 is supported by the recent findings of Nozuma et al. demonstrating a significant association between TRIM5 R136Q polymorphism and lower PVL, specific to HAM/TSP patients (Nozuma et al., 2017). Strikingly, polymorphisms in TRIM5, TRIM22, and BST2, but not APOBECs or TREX1 were significantly associated to another neuroinflammatory disorder, multiple sclerosis (Nexo et al., 2013). Conversely, the apparent proviral effect of PAF-1, ISG15, and CDKN1A are in agreement with the recently demonstrated IFN-inducible HAM/TSP disease signature (Tattermusch et al., 2012). Moreover, the robustness of the antiviral and proviral clusters we identified in this study indicates they represent plausible candidate biomarkers for future HAM/TSP clinical trials. In agreement with Saito et al., who demonstrated a positive correlation between HBZ mRNA levels and HAM/TSP disease severity (Saito et al., 2009), our cluster analysis shows the strongest association between $\mathrm{HBZ}$ and clinical status 

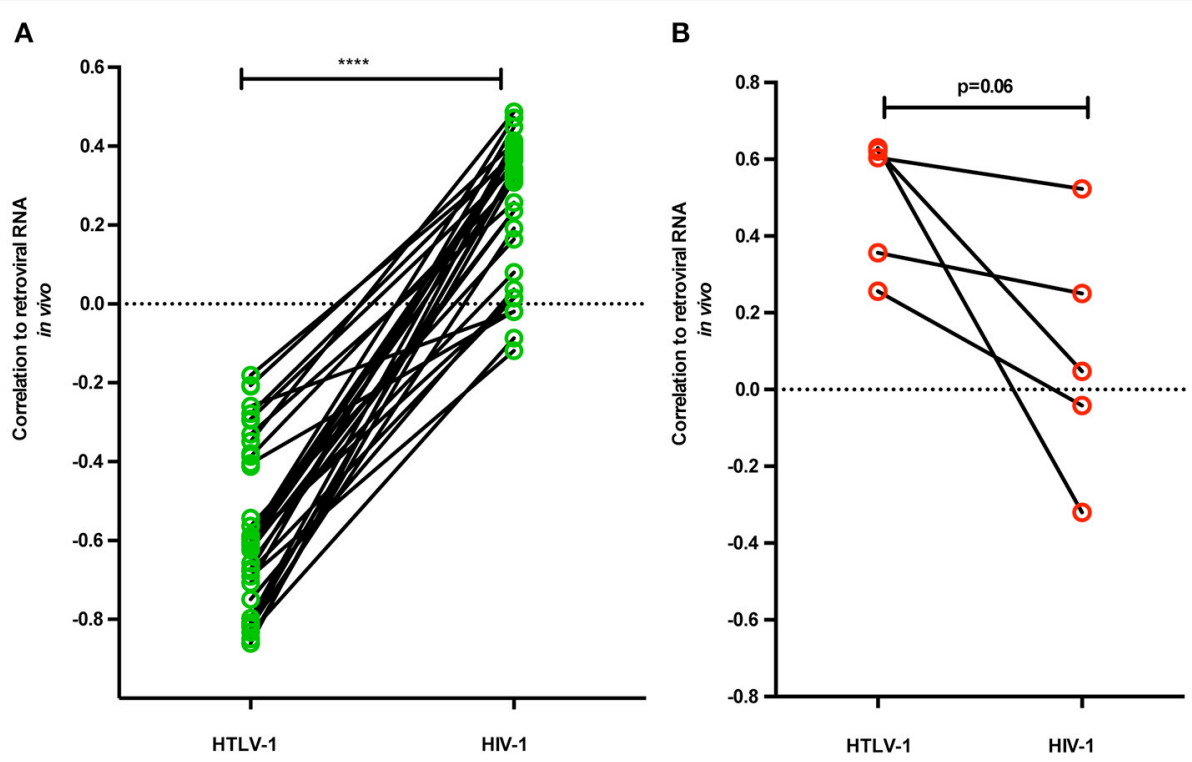

FIGURE 4 | Restriction factors correlate oppositely to in vivo retroviral RNA levels in untreated HTLV-1 vs. HIV-1 infection. A pairwise analysis was performed for RFs in antiviral and proviral clusters, by correlating to in vivo RNA levels in both retroviral infections, using HTLV-1 data from this study (Figure 1A) and data from the Swiss HIV cohort (Rotger et al., 2010). Out of 42 RFs, 40 were matched to unique transcripts in HIV-1 microarray data, followed by calculating transcriptome-wide correlation of viral load set point to each separate gene, using stringent FDR (Benjamini-Hochberg) correction. (A) HTLV-1 "antiviral" cluster genes show significantly increased (positive) correlation to HIV-1 in vivo RNA levels ( ${ }^{\star \star \star \star} p<0.0001$, Wilcoxon signed rank test, $\left.n=35\right)$. (B) HTLV-1 "proviral" cluster genes show a tendency for decreased (positive) correlation to HIV-1 in vivo RNA levels ( $p=0.06$, Wilcoxon signed rank test, $n=5)$.

(Figure 1A). However, no RFs were significantly correlated to HBZ mRNA levels after correction for multiple testing, in contrast to tax mRNA levels (Figure 1B). This might be due to post-transcriptional regulation of $\mathrm{HBZ}$ mRNA vs. protein levels, as Baratella et al. recently demonstrated cytoplasmatic HBZ protein as a biomarker able to discriminate between AC and HAM/TSP (Baratella et al., 2017). Due to their significant association with all currently used surrogate markers (PVL, Tax, and $H B Z$ mRNAs), as well as clinical status, antiviral and proviral clusters are likely to reflect positive vs. negative therapeutic outcomes, respectively. Therefore, our results help explain the "IFN paradox" in HAM/TSP, by reconciling apparently conflicting data in the literature, namely the existence of an interferon-inducible disease signature and the demonstrated beneficial therapeutic effects of type I interferons, both IFN- $\alpha$ and $\beta$ in HAM/TSP (Izumo et al., 1996; Oh et al., 2005).

In addition, our study also reveals striking differences between these two widely used IFN subtypes. First, a significant antiproliferative effect of $\beta$ and not $\alpha$ was observed in HAM/TSP patients, corroborating our recent findings in ATL (Dierckx et al., 2017) and revealing a surprising similarity between these two highly distinct HTLV-1-associated pathologies. On the other hand, our study also reveals another parallel between neuroinflammatory disorders HAM/TSP and multiple sclerosis, namely their unique sensitivity to IFN- $\beta$ over IFN- $\alpha$. Of note, our results provide mechanistic evidence for the previously described immunovirological and clinical impact observed in vivo with IFN- $\beta$ therapy in HAM/TSP patients, in an open-label trial (Oh et al., 2005), as well as two case reports with remarkable clinical response (Costa et al., 2012; Viana et al., 2014). This superior antiproliferative effect of IFN- $\beta$ in HAM/TSP, as compared to IFN- $\alpha$, parallels our findings in the other major HTLV-1associated disease, Adult T-cell Leukemia (Dierckx et al., 2017).

Surprisingly, the "proviral/deleterious" gene cluster, identified by its strong positive correlation to HTLV-1 RNA levels and PVL, contains the RFs previously demonstrated to exert the strongest protective anti-HIV-1 effect in vivo and in vitro. Thus, p21 (CDKN1A), schlafen 11 (SLFN11), and PAF1 were strongly associated with reduced CD4+ $\mathrm{T}$ cell-associated HIV RNA during antiretroviral treatment (Abdel-Mohsen et al., 2015). SLFN11 was also identified as overexpressed in HIV-positive "elite controllers" (Abdel-Mohsen et al., 2013), who maintain undetectable viral load, even in the absence of antiretroviral treatment. PAF1 was identified by a genome-wide screen for HIV RFs (Liu et al., 2011) and restricts HIV-1, HIV-2, and SIV in vitro. Finally, CDKN1A was demonstrated as a robust antiviral effect against HIV-1 replication in vitro, by different groups (Leng et al., 2014; Farberov et al., 2015).

With up to eight percent of the human genome of retroviral origin, the importance of retroviruses for the evolution of susceptible host organisms cannot be overestimated (Ortiz et al., 2009; Lascano et al., 2015). Over millions of years, Lentivirus infections (retroviruses including HIV and ancestral SIV) have had a pronounced effect upon primate evolution (Ortiz et al., 2009). Yet, no evidence exists for deltaretroviruses (including HTLV and PTLV). Using two different quantitative approaches, i.e., measuring chimp/human cross-species adaptive evolution (Figures 5A,B) and purifying selection throughout primate 
A

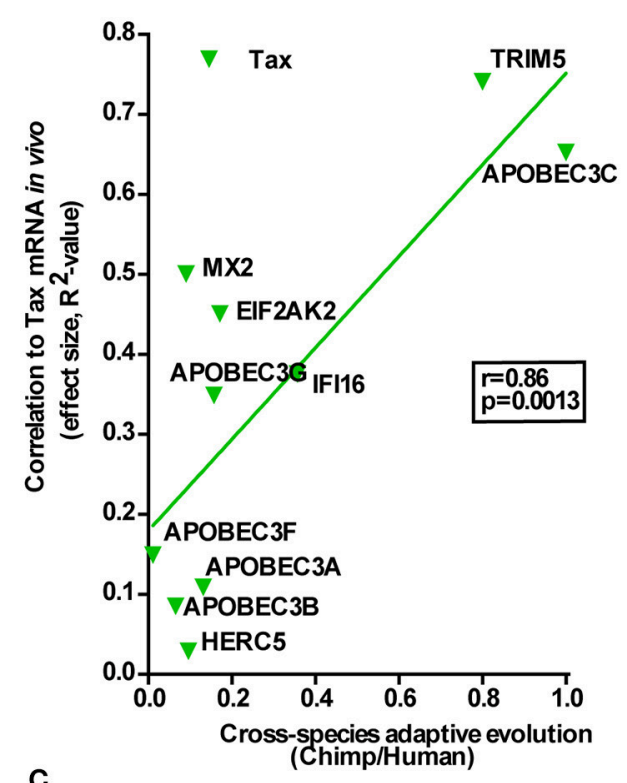

。

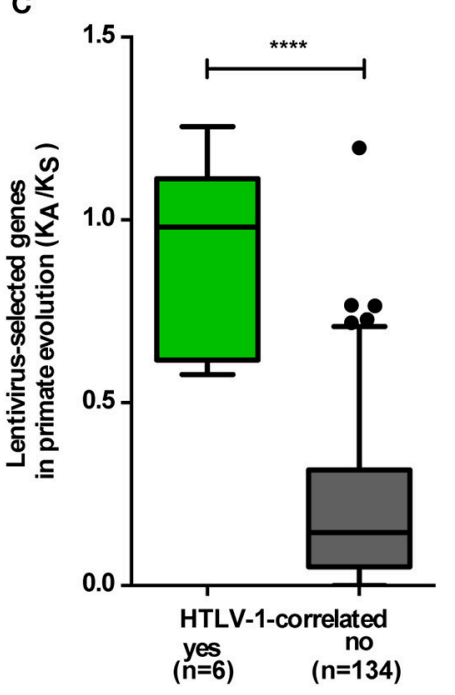

B

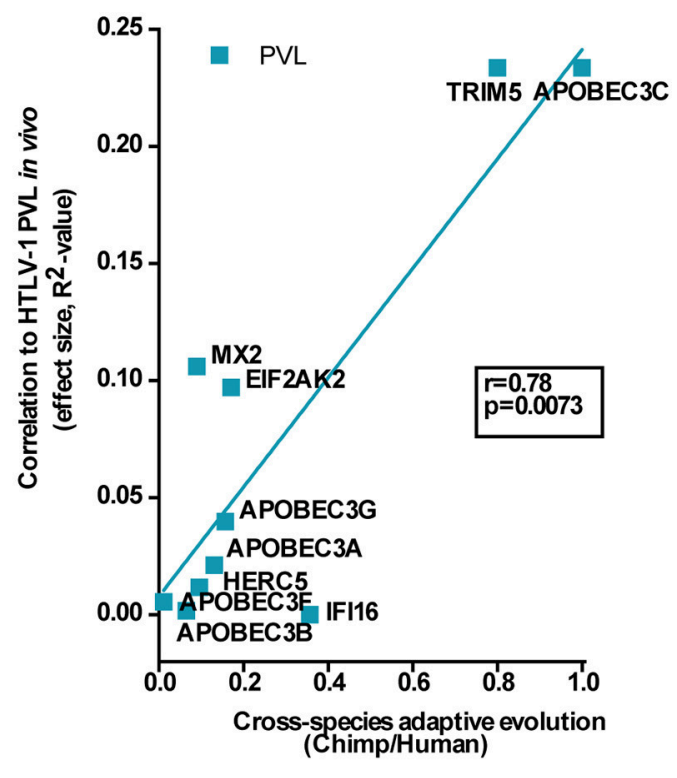

FIGURE 5 | Lentivirus-driven primate evolution has shaped antiretroviral activity in untreated HTLV-1 infection. The antiviral RF cluster is strongly selected during recent primate evolution, as measured by both cross-species adaptive evolution between chimpanzees and humans and purifying selection throughout primate evolution. (A,B) Among the 42 anti-HIV RFs selected in this study, 10 were found to display chimpanzee/human cross-species adaptive evolution (McLaren et al., 2015). The anti-HTLV-1 effect (measured as $R^{2}$ ) of these 10 genes was significantly correlated to their adaptive evolution score, for both Tax mRNA $(r=0.86, p=$ $0.0013)$ and PVL ( $r=0.78, p=0.0073)$. (C) Six genes of the proposed antiviral cluster underwent lentivirus-driven purifying selection (measured as $\mathrm{K}_{\mathrm{A}} / \mathrm{K}_{\mathrm{S}}$, Ortiz et al., 2009) during primate evolution, which was significantly increased vs. all other lentivirus-selected primate genes $\left(n=134\right.$, ${ }^{* * \star *} p<0.0001$, Mann-Whitney test).

evolution (Figure 5C), we found that the antiviral RF cluster we identified for HTLV-1 was under strong purifying selection during recent primate evolution. Our preselection of a small number of candidate RFs, by virtue of their proven antiviral effect against HIV-1, is an obvious limitation of the first part of our study, focusing on purified CD4+ cells. However, we confirm the unique TRIM5/TRIM22/BST2 antiviral cluster by two independent unbiased methods, i.e., PBMC transcriptomics and genome-wide evolutionary analysis of protein-coding genes. By comparing the only two pathogenic human retroviruses, we found striking differences in in vivo correlations of "candidate" RFs to retroviral RNA levels, as an indirect measure of their possible anti-HIV and anti-HTLV-1 activity. Although some antiviral effector molecules such as cGAS exhibit "panviral" activity (Schoggins et al., 2014), our results argue against "panretroviral" restriction factors in the human setting. Very few host genes, such as CIITA and PKR (also known as EIF2AK2), have been formally demonstrated to restrict both HIV-1 and HTLV-1 replication in vitro (Schoggins et al., 2011; Tosi et al., 2011; Cachat et al., 2013; Kinpara et al., 2013; 
Forlani et al., 2016). Interestingly, CIITA interacts and cooperates with TRIM22 in restricting replication of HIV-1 (Forlani et al., 2017). However, CIITA is unique among RFs because it is transcriptionally upregulated by IFN-gamma but downregulated by IFN- $\beta$ (Lu et al., 1995). In contrast, IFN- $\alpha$ strongly upregulates PKR/EIF2AK2 in HTLV-1-infected cells but does not decrease Tax or Hbz mRNA (Moens et al., 2012b; Cachat et al., 2013; Kinpara et al., 2013). Thus, CIITA and PKR are unlikely candidates for the IFN- $\beta$-mediated antiviral and antiproliferative effect we observed ex vivo and in vitro in HAM/TSP patients. Unfortunately, high-throughput in vitro analysis of RFs, such as performed by Schoggins et al. for HIV-1 and several other viruses, are lacking for HTLV-1 (Schoggins et al., 2011). Based on our previous demonstration of a 1000-fold difference in HIV-1 vs. HTLV-1 antiviral effect in co-infected MT-4 cells (Moens et al., 2012b) we anticipate strong differences might be observed for in vitro HTLV-1 vs. HIV-1 restriction pathways. As a final layer of complexity, HIV-1 also displays striking discrepancies between in vivo and ex vivo RF antiviral activity (Rotger et al., 2010; Schoggins et al., 2014). Therefore, we believe the term "restriction factors" should be used with caution in human retrovirology. "Antiviral effector genes" might be a more precise term, if defined within a precise context, i.e., depending on cell type, virus and upstream regulators such as IFN subtypes. Regarding cell typespecificity, TRIM5 $\alpha$ and TRIM22 expression at both mRNA and protein level has been shown to predominate in $\mathrm{CD} 4^{+} \mathrm{T}$ cells, as compared to other mononuclear cell types (Singh et al., 2014), which explains the strong agreement between our RF analysis in $\mathrm{CD}^{+}{ }^{+} \mathrm{T}$ cells and IFN response in PBMCs, as well as whole blood analysis from UK cohort participants (Tattermusch et al., 2012).

Mechanistically, both the antiviral vs. "proviral" effects of TRIM5, CDKN1A, and ISG15 might also be explained by broader cellular processes, outside the narrow definition of RFs. TRIM5 is an intracellular protein that exerts its protective effect by disrupting the retroviral capsid as it transports viral nucleic acid into the nucleus. In addition, innate immune signaling might contribute to TRIM5-mediated restriction. Lascano et al. have shown that activation of innate immune signaling is conserved among primate and carnivore TRIM5 orthologs and that such activity is required for TRIM5mediated restriction activity (Lascano et al., 2015). CDKN1A upregulation by Tax might shorten G1 phase by promoting formation of stable kinase complexes, contributing to cellcycle progression (Kehn et al., 2004) and proliferation of infected clones. Regarding ISG15, our group has recently demonstrated its anti-inflammatory extracellular cytokine-like activity, through monocyte-specific induction of IL-10 (Dos

\section{REFERENCES}

Abdel-Mohsen, M., Raposo, R. A., Deng, X., Li, M., Liegler, T., Sinclair, E., et al. (2013). Expression profile of host restriction factors in HIV-1 elite controllers. Retrovirology 10:106. doi: 10.1186/1742-4690-10-106

Abdel-Mohsen, M., Wang, C., Strain, M. C., Lada, S. M., Deng, X., Cockerham, L. R., et al. (2015). Select host restriction factors are associated
Santos et al., 2018). However, recent data reveal an unexpected IL-10-induced proliferative switch in HAM/TSP-derived HTLV1-infected T-cell lines (Sawada et al., 2017). Therefore, ISG15 inducing IL-10 and hence triggering lymphoproliferation might represent, at least in part, a molecular mechanism for the putative "proviral" role of ISG15 in HAM/TSP. Integrating the data from Tattermusch et al. (2012), Dos Santos et al. (2018), Sawada et al. (2017) and this study, we hypothesize that an "IFN-beta like/TRIM5" gene signature vs. a "nonIFN-beta like/ISG15" gene signature might be predictive of HAM/TSP disease progression, as well as of therapeutic outcome with immunomodulatory and/or antiproliferative drugs (IFNs, glucocorticoids and others).

In conclusion, our integrated ex vivo approach reveals that antiretroviral genes in HTLV-1 infection and HAM/TSP cluster in two distinct "proviral/antiviral" classes, of which the TRIM5 $\alpha /$ TRIM22/BST2 antiviral subset, selected during recent primate evolution, is selectively up-regulated by IFN- $\beta$ signaling in HAM/TSP. Our results thus provide ex vivo mechanistic evidence for the observed in vivo immunovirological effect of IFN- $\beta$ treatment in HAM/TSP and identify biomarkers as well as possible therapeutic targets for a precision medicine approach. Finally, a protective antiviral and IFN-inducible TRIM $5 \alpha /$ TRIM22/BST2 gene cluster, independent of the HAM/TSP IFN-inducible disease signature, reconciles the apparent IFN paradox in HTLV-1 research and confirms type I IFN as a two-edged sword in human health and disease.

\section{AUTHOR CONTRIBUTIONS}

FL, RR, and JVW: conceived and designed the experiments; SM, FL, EC, PB, and JVW: performed the experiments; FL, SM, LG, RR, RK, JV, TD, and JVW: analyzed the data; EK, DN, AS, BG-C, and LG: contributed reagents, materials, and analysis tools; FL, SM, JVW, and DN: wrote the paper.

\section{FUNDING}

This work was supported by $\mathrm{CNPq}$ (Pronex, PVE), KU Leuven (Vaast Leysen Leerstoel voor Infectieziekten in Ontwikkelingslanden) and FWO (grant G0D6817N). This work was partially supported by the National Institutes of Health grant to the District of Columbia Center for AIDS Research, (P30AI087714 and P30AI117970), Fundação de Amparo a Pesquisa do Estado de São Paulo (04/15856-9/EGK and 2010/05845-0/EGK/DFN), CNPq/CAPES 056/2012 (DFN). 
HTLV-1-associated myelopathy/tropical spastic paraparesis (HAM/TSP). PLoS Negl. Trop. Dis. 11:e0005285. doi: 10.1371/journal.pntd.0005285

Cachat, A., Chevalier, S. A., Alais, S., Ko, N. L., Ratner, L., Journo, C., et al. (2013). Alpha interferon restricts human T-lymphotropic virus type 1 and 2 de novo infection through PKR activation. J. Virol. 87, 13386-13396. doi: 10.1128/JVI.02758-13

Costa, D. T., Sundberg, M., Passos, L., Muniz, A. L., and Santos, S. (2012). Interferon Beta-1a improves urinary symptoms, reduces proviral load, and modifies the immune response in a patient with HAM/TSP. Case Rep. Neurol. Med. 2012:958786. doi: 10.1155/2012/958786

Dehee, A., Cesaire, R., Desire, N., Lezin, A., Bourdonne, O., Bera, O., et al. (2002). Quantitation of HTLV-I proviral load by a TaqMan real-time PCR assay. J. Virol. Methods 102, 37-51. doi: 10.1016/S0166-0934(01)00445-1

Dierckx, T., Khouri, R., Menezes, S. M., Decanine, D., Farre, L., Bittencourt, A., et al. (2017). IFN- $\beta$ induces greater antiproliferative and proapoptotic effects and increased p53 signaling compared with IFN- $\alpha$ in PBMCs of Adult T-cell Leukemia/Lymphoma patients. Blood Cancer J. 7:e519. doi: $10.1038 /$ bcj.2016.126

Dos Santos, P. F., Van Weyenbergh, J., Delgobo, M., Oliveira Patricio, D., Ferguson, B. J., Guabiraba, R., et al. (2018). ISG15-induced IL-10 Is a novel antiinflammatory myeloid axis disrupted during active tuberculosis. J. Immunol. 200, 1434-1442. doi: 10.4049/jimmunol.1701120

Farberov, L., Herzig, E., Modai, S., Isakov, O., Hizi, A., and Shomron, N. (2015). MicroRNA-mediated regulation of p21 and TASK1 cellular restriction factors enhances HIV-1 infection. J. Cell Sci. 128, 1607-1616. doi: 10.1242/jcs.167817

Forlani, G., Tosi, G., Turrini, F., Poli, G., Vicenzi, E., and Accolla, R. S. (2017). Tripartite motif-containing protein 22 interacts with class II transactivator and orchestrates its recruitment in nuclear bodies containing TRIM19/PML and cyclin T1. Front. Immunol. 8:564. doi: 10.3389/fimmu.2017.00564

Forlani, G., Turrini, F., Ghezzi, S., Tedeschi, A., Poli, G., Accolla, R. S., et al. (2016). The MHC-II transactivator CIITA inhibits Tat function and HIV-1 replication in human myeloid cells. J. Transl. Med. 14:94. doi: 10.1186/s12967-016-0853-5

Foster, T. L., Pickering, S., and Neil, S. J. D. (2017). Inhibiting the ins and outs of HIV replication: cell-intrinsic antiretroviral restrictions at the plasma membrane. Front. Immunol. 8:1853. doi: 10.3389/fimmu.2017.01853

Gallo, R. C., Willems, L., and Tagaya, Y. (2017). Time to Go back to the original name. Front. Microbiol. 8:1800. doi: 10.3389/fmicb.2017.01800

Izumo, S., Goto, I., Itoyama, Y., Okajima, T., Watanabe, S., Kuroda, Y., et al. (1996). Interferon-alpha is effective in HTLV-I-associated myelopathy: a multicenter, randomized, double-blind, controlled trial. Neurology 46,1016-1021. doi: 10.1212/WNL.46.4.1016

Kehn, K., Deng, L., de la Fuente, C., Strouss, K., Wu, K., Maddukuri, A., et al. (2004). The role of cyclin D2 and p21/waf1 in human T-cell leukemia virus type 1 infected cells. Retrovirology 1:6. doi: 10.1186/1742-4690-1-6

Kinpara, S., Kijiyama, M., Takamori, A., Hasegawa, A., Sasada, A., Masuda, T., et al. (2013). Interferon- $\alpha$ (IFN- $\alpha$ ) suppresses HTLV-1 gene expression and cell cycling, while IFN- $\alpha$ combined with zidovudine induces p53 signaling and apoptosis in HTLV-1-infected cells. Retrovirology 10:52. doi: 10.1186/1742-4690-10-52

Lascano, J., Uchil, P. D., Mothes, W., and Luban, J. (2015). TRIM5 retroviral restriction activity correlates with the ability to induce innate immune signaling. J. Virol. 90, 308-316. doi: 10.1128/JVI.02496-15

Leng, J., Ho, H. P., Buzon, M. J., Pereyra, F., Walker, B. D., Yu, X. G., et al. (2014). A cell-intrinsic inhibitor of HIV-1 reverse transcription in CD4(+) T cells from elite controllers. Cell Host Microbe 15, 717-728. doi: 10.1016/j.chom.2014.05.011

Liu, L., Oliveira, N. M., Cheney, K. M., Pade, C., Dreja, H., Bergin, A. M., et al. (2011). A whole genome screen for HIV restriction factors. Retrovirology 8:94. doi: $10.1186 / 1742-4690-8-94$

Lu, H. T., Riley, J. L., Babcock, G. T., Huston, M., Stark, G. R., Boss, J. M., et al. (1995). Interferon (IFN) beta acts downstream of IFN-gamma-induced class II transactivator messenger RNA accumulation to block major histocompatibility complex class II gene expression and requires the 48 -kD DNA-binding protein, ISGF3-gamma. J. Exp. Med. 182, 1517-1525. doi: 10.1084/jem.182.5.1517

McLaren, P. J., Gawanbacht, A., Pyndiah, N., Krapp, C., Hotter, D., Kluge, S. F., et al. (2015). Identification of potential HIV restriction factors by combining evolutionary genomic signatures with functional analyses. Retrovirology 12:41. doi: $10.1186 / \mathrm{s} 12977-015-0165-5$
Menezes, S. M., Decanine, D., Brassat, D., Khouri, R., Schnitman, S. V., Kruschewsky, R., et al. (2014). CD80+ and CD86+ B cells as biomarkers and possible therapeutic targets in HTLV-1 associated myelopathy/tropical spastic paraparesis and multiple sclerosis. J. Neuroinflammation 11:18. doi: 10.1186/1742-2094-11-18

Moens, B., Decanine, D., Menezes, S. M., Khouri, R., Silva-Santos, G., Lopez, G., et al. (2012a). Ascorbic acid has superior ex vivo antiproliferative, cell deathinducing and immunomodulatory effects over IFN- $\alpha$ in HTLV-1-associated myelopathy. PLoS Negl. Trop. Dis 6:e1729. doi: 10.1371/journal.pntd.0001729

Moens, B., Pannecouque, C., Lopez, G., Talledo, M., Gotuzzo, E., Khouri, R., et al. (2012b). Simultaneous RNA quantification of human and retroviral genomes reveals intact interferon signaling in HTLV-1-infected CD4+ T cell lines. Virol. J. 9:171. doi: 10.1186/1743-422X-9-171

Neil, S. J., Zang, T., and Bieniasz, P. D. (2008). Tetherin inhibits retrovirus release and is antagonized by HIV-1 Vpu. Nature 451, 425-430. doi: 10.1038 /nature 06553

Nexo, B. A., Hansen, B., Nissen, K. K., Gundestrup, L., Terkelsen, T., Villesen, P., et al. (2013). Restriction genes for retroviruses influence the risk of multiple sclerosis. PLoS ONE 8:e74063. doi: 10.1371/journal.pone.0074063

Nozuma, S., Matsuura, E., Kodama, D., Tashiro, Y., Matsuzaki, T., Kubota, R., et al. (2017). Effects of host restriction factors and the HTLV-1 subtype on susceptibility to HTLV-1-associated myelopathy/tropical spastic paraparesis. Retrovirology 14:26. doi: 10.1186/s12977-017-0350-9

Oh, U., Yamano, Y., Mora, C. A., Ohayon, J., Bagnato, F., Butman, J. A., et al. (2005). Interferon-betala therapy in human T-lymphotropic virus type I-associated neurologic disease. Ann. Neurol. 57, 526-534. doi: 10.1002/ana.20429

Ortiz, M., Guex, N., Patin, E., Martin, O., Xenarios, I., Ciuff, A., et al. (2009). Evolutionary trajectories of primate genes involved in HIV pathogenesis. Mol. Biol. Evol. 26, 2865-2875. doi: 10.1093/molbev/msp197

Osame, M. (1990). "Review of WHO Kagoshima Meeting and diagnostic guidelines for HAM/TSP," in Human Retrovirology: HTLV, ed W. Blattner (New York, NY: Raven Press), 191-177.

Osame, M., Usuku, K., Izumo, S., Ijichi, N., Amitani, H., Igata, A., et al. (1986). HTLV-I associated myelopathy, a new clinical entity. Lancet 1, 1031-1032. doi: 10.1016/S0140-6736(86)91298-5

Raposo, R. A., Abdel-Mohsen, M., Bilska, M., Montefiori, D. C., Nixon, D. F., and Pillai, S. K. (2013a). Effects of cellular activation on anti-HIV-1 restriction factor expression profile in primary cells. J. Virol. 87, 11924-11929. doi: 10.1128/JVI.02128-13

Raposo, R. A., Abdel-Mohsen, M., Deng, X., Hecht, F. M., Pilcher, C. D., Pillai, S. K., et al. (2014). Dynamic regulation of host restriction factor expression over the course of HIV-1 infection in vivo. J. Virol. 88, 11624-11629. doi: 10.1128/JVI.01771-14

Raposo, R. A., Abdel-Mohsen, M., Holditch, S. J., Kuebler, P. J., Cheng, R. G., Eriksson, E. M., et al. (2013b). Increased expression of intrinsic antiviral genes in HLA-B*57-positive individuals. J. Leukoc. Biol. 94, 1051-1059. doi: $10.1189 / \mathrm{jlb} .0313150$

Rotger, M., Dang, K. K., Fellay, J., Heinzen, E. L., Feng, S., Descombes, P., et al. (2010). Genome-wide mRNA expression correlates of viral control in CD4+ T-cells from HIV-1-infected individuals. PLoS Pathog. 6:e1000781. doi: 10.1371/journal.ppat.1000781

Saito, M., Matsuzaki, T., Satou, Y., Yasunaga, J., Saito, K., Arimura, K., et al. (2009). In vivo expression of the HBZ gene of HTLV-1 correlates with proviral load, inflammatory markers and disease severity in HTLV-1 associated myelopathy/tropical spastic paraparesis (HAM/TSP). Retrovirology 6:19. doi: 10.1186/1742-4690-6-19

Sawada, L., Nagano, Y., Hasegawa, A., Kanai, H., Nogami, K., Ito, S., et al. (2017). IL-10-mediated signals act as a switch for lymphoproliferation in Human T-cell leukemia virus type-1 infection by activating the STAT3 and IRF4 pathways. PLoS Pathog. 13:e1006597. doi: 10.1371/journal.ppat.1006597

Schoggins, J. W., MacDuff, D. A., Imanaka, N., Gainey, M. D., Shrestha, B., Eitson, J. L., et al. (2014). Pan-viral specificity of IFN-induced genes reveals new roles for cGAS in innate immunity. Nature 505, 691-695. doi: 10.1038/nature 12862

Schoggins, J. W., Wilson, S. J., Panis, M., Murphy, M. Y., Jones, C. T., Bieniasz, P., et al. (2011). A diverse range of gene products are effectors of the type I interferon antiviral response. Nature 472, 481-485. doi: 10.1038/nature09907 
Singh, R., Patel, V., Mureithi, M. W., Naranbhai, V., Ramsuran, D., Tulsi, S., et al. (2014). TRIM5alpha and TRIM22 are differentially regulated according to HIV-1 infection phase and compartment. J. Virol. 88, 4291-4303. doi: $10.1128 /$ JVI.03603-13

Tagaya, Y., and Gallo, R. C. (2017). The exceptional oncogenicity of HTLV-1. Front. Microbiol. 8:1425. doi: 10.3389/fmicb.2017.01425

Tattermusch, S., Skinner, J. A., Chaussabel, D., Banchereau, J., Berry, M. P., McNab, F. W., et al. (2012). Systems biology approaches reveal a specific interferon-inducible signature in HTLV-1 associated myelopathy. PLoS Pathog. 8:e1002480. doi: 10.1371/journal.ppat.1002480

Telenti, A. (2005). Adaptation, co-evolution, and human susceptibility to HIV-1 infection. Infect. Genet. Evol. 5, 327-334. doi: 10.1016/j.meegid.2004.11.001

Tosi, G., Forlani, G., Andresen, V., Turci, M., Bertazzoni, U., Franchini, G., et al. (2011). Major histocompatibility complex class II transactivator CIITA is a viral restriction factor that targets human T-cell lymphotropic virus type 1 Tax-1 function and inhibits viral replication. J. Virol. 85, 10719-10729. doi: 10.1128/JVI.00813-11
Viana, G. M., Silva, M. A., Souza, V. L., Lopes, N. B., Silva, D. L., and Nascimento Mdo, D. (2014). Interferon beta-la treatment in HTLV-1associated myelopathy/tropical spastic paraparesis: a case report. Rev. Inst. Med. Trop. Sao Paulo 56, 443-445. doi: 10.1590/S0036-46652014000500013

Conflict of Interest Statement: The authors declare that the research was conducted in the absence of any commercial or financial relationships that could be construed as a potential conflict of interest.

Copyright (๑) 2018 Leal, Menezes, Costa, Brailey, Gama, Segurado, Kallas, Nixon, Dierckx, Khouri, Vercauteren, Galvão-Castro, Raposo and Van Weyenbergh. This is an open-access article distributed under the terms of the Creative Commons Attribution License (CC BY). The use, distribution or reproduction in other forums is permitted, provided the original author(s) and the copyright owner are credited and that the original publication in this journal is cited, in accordance with accepted academic practice. No use, distribution or reproduction is permitted which does not comply with these terms. 